\title{
Spectral Analysis of Arterial Blood Pressure Variability: Assessing the Accuracy of the Finometer Device
}

\author{
R Maestri, GD Pinna, E Gobbi, E Robbi, MT La Rovere
}

\author{
S. Maugeri Foundation, Scientific Institute of Montescano, Montescano (PV), Italy
}

\begin{abstract}
In this study we appraised the accuracy of the Finometer in spectral analysis of systolic (SAP) and diastolic (DAP) arterial pressure. In 24 patients the blood pressure signal was simultaneously recorded invasively and by the Finometer and the power of SAP and DAP in the VLF $(0.01 \div 0.04 \mathrm{~Hz}), L F(0.04 \div 0.15 \mathrm{~Hz})$, and $\mathrm{HF}(0.15 \div 0.45 \mathrm{~Hz})$ bands computed. To investigate the relationship between invasive and Finometer measurements, the percentage error was computed and bivariate spectral analysis performed. The error for SAP analysis, was (\%): 139, 76, -32, 75 for respectively $V L F$, $L F, H F$ and total power; DAP errors were much smaller.

$S A P$ variability is greatly enhanced by the Finometer, mainly due to the increase of power in the VLF and LF bands which overrides the reduction in the HF band. Noise and nonlinearities account for $<20 \%$ of the Finometer power changes, indicating that the differences are mainly due to amplification or attenuation effects.
\end{abstract}

\section{Introduction}

In the last years a large number of researchers involved in the investigation of the neural control of the cardiovascular function have analyzed the spectral content of beat-by-beat arterial blood pressure fluctuations recorded using the Finapres monitor, a noninvasive device based on the Penaz method. This device, whose accuracy has been widely investigated [1, 2], has been recently replaced by the Finometer equipment (FMS, Finapres Medical Systems BV, Arnhem, the Netherlands). Since this new device is likely going to be used in many studies, we devised the present investigation to appraise its accuracy in spectral analysis of systolic (SAP) and diastolic (DAP) arterial pressure, comparing observed measurements to those simultaneously obtained by intra-arterial cannulation. To identify and better understand the relationship between the invasive and noninvasive pressure signals, we performed linear system analysis, taking into consideration a mixed population of cardiac disease subjects, namely post-myocardial infarction, chronic heart failure patients and cardiac transplant patients, which are of frequent interest in cardiovascular variability studies.

\section{Methods}

\subsection{Study population}

Twenty-four patients (mean age 56, range 41-76 years) participated in the study. Of them, 6 were post infarct (post-MI) patients, 7 were cardiac transplant (CT) and 11 were heart failure (HF) patients. Subjects were excluded from the study if they had an arterial systolic blood pressure $>160 \mathrm{mmHg}$ and/or a diastolic blood pressure $>$ $90 \mathrm{mmHg}$, atrial fibrillation or abnormal sinus node function. All patients gave informed consent to participation in the study.

\subsection{Signals and experimental protocol}

All patients carried out a resting recording of ECG and arterial blood pressure in our laboratory for the investigation of the autonomic nervous system. The arterial blood pressure signal was simultaneously recorded invasively via brachial artery cannulation and noninvasively via the Finometer device. The invasive measurement was obtained by an Abbocath-T22G teflon cannula connected to a Ohmeda P23XL-1 pressure transducer. The Finometer pressure signal was acquired from the analog output of the device. During recording, patients lay down in the supine position in a quiet dim room. After instrumentation, calibration and proper signal stabilization (15 $\mathrm{min}$ ), the Finometer self adjustment was disconnected and an 8 minute recording of ECG, Finometer and invasive arterial blood pressure was performed. All analog signals were digitized at a sampling frequency of $250 \mathrm{~Hz}$ and acquired on a personal computer. From the ECG we derived the time series of R$\mathrm{R}$ intervals. Beat-by-beat systolic and diastolic pressure values were measured for each RR interval respectively as the maximum and minimum of the pressure curve in the same interval. All raw time series of invasive and noninvasive systolic and diastolic pressure were visually 
inspected to select a sample record free from large transients and artefacts in all signals. Some editing facilities were set up to correct for ectopic beats. Recordings having an ectopy rate $>2 \%$ were excluded from the analysis. A linear detrending algorithm, based on a first order least square polynomial fitting, was applied to all sample records before analysis.
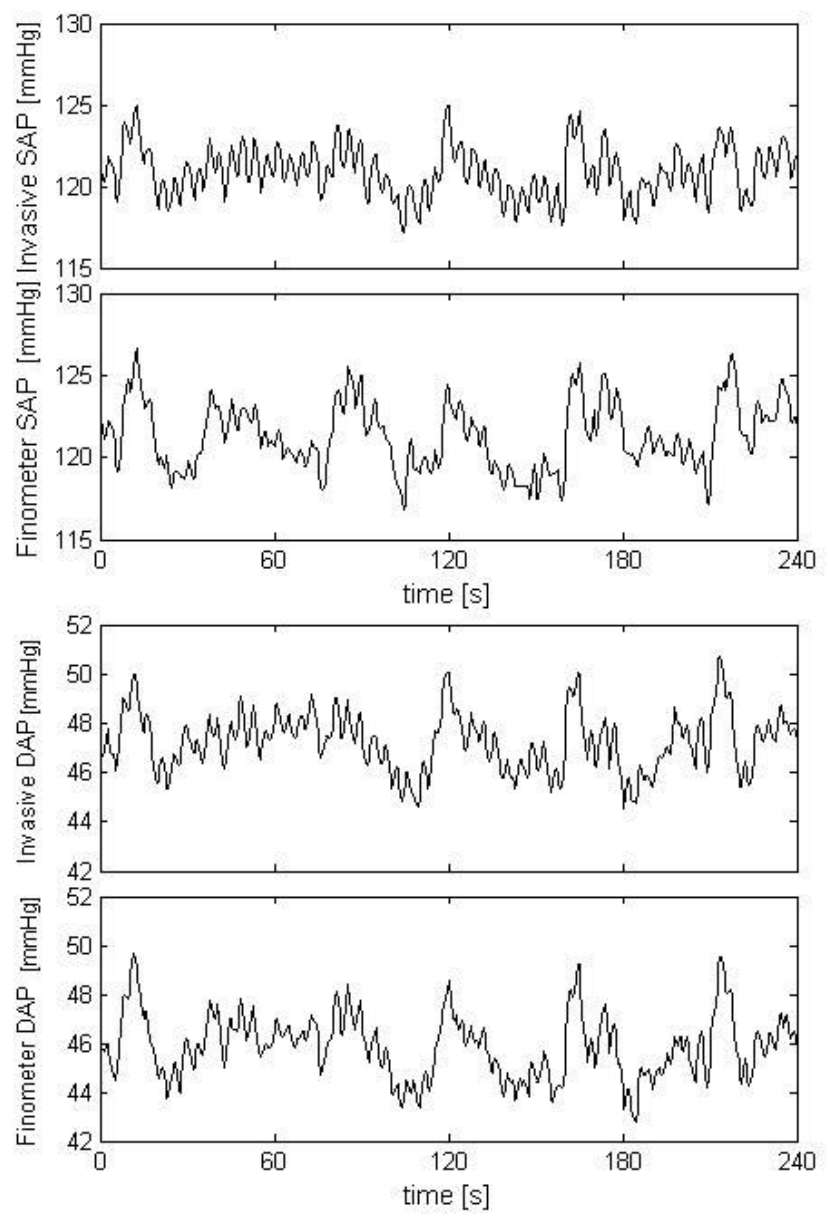

Figure 1. From top to bottom: recording of Invasive SAP, Finometer SAP, Invasive DAP and Finomter DAP in a 43 years old post-MI patient.

\subsection{Signal processing}

The power spectral density of each invasive and noninvasive pressure sequence was estimated by the Blackman-Tukey method, that is by FFT transforming the windowed sample autocovariance function of the data. The spectral window we used was the one proposed by Parzen with a spectral bandwidth of $0.015 \mathrm{~Hz}$ [3]. According to the recommendations of the Task Force [4], the signal bandwidth was subdivided into 3 frequency regions: 0.01-0.04 Hz (Very Low Frequency, VLF), 0.04$0.15 \mathrm{~Hz}$ (Low Frequency, LF), 0.15-0.45 Hz (High
Frequency, HF). The power in each band was computed by numerical integration. The accuracy of noninvasive power measurements was expressed as percentage error relative to the invasive ones. To investigate the relationship between invasive and Finometer measurements we performed bivariate spectral analysis and computed, in each band, the percentage of power in the Finometer signal linearly related to the invasive signal (coherent output power, COP). We also computed the average coherence and the average transfer function in the LF band between SAP (both invasive and noninvasive) and RR time series. This latter parameter is of particular practical interest since it has been proposed as a method to assess baroreflex sensitivity [5]. The population of transplanted patients was excluded from this part of the analysis, since their heart is denervated and almost unaffected by the baroreceptors reflexes.

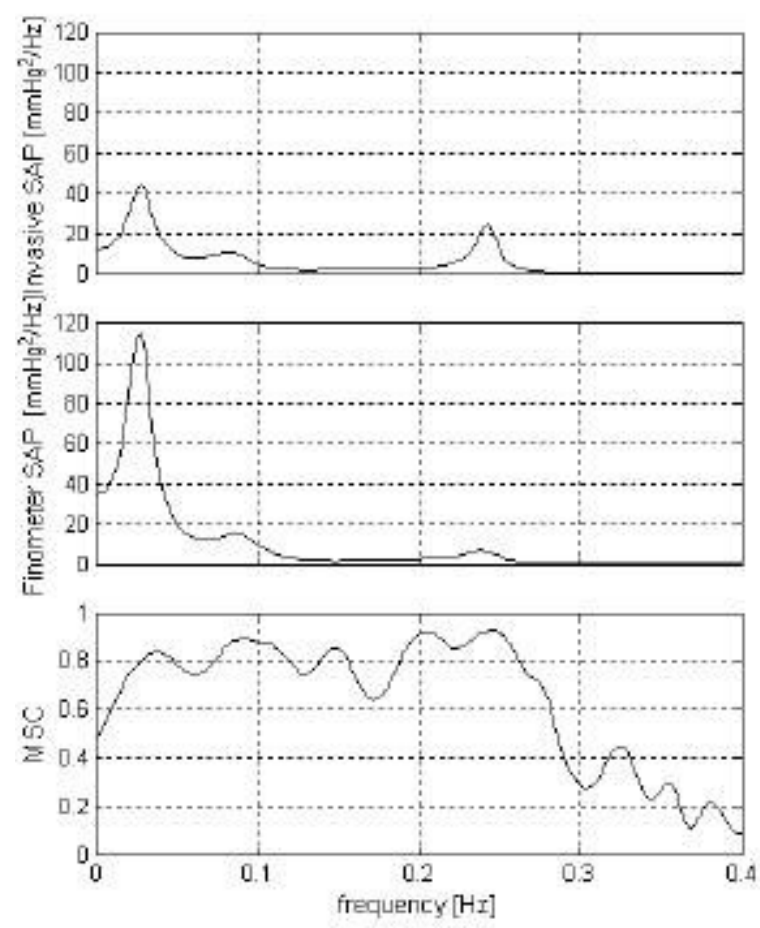

Figure 2. From top to bottom: Power spectral density of Invasive SAP, power spectral density of Finometer SAP and magnitude square coherence (MSC) function between the two signals in the same patient of Figure 1.

\subsection{Data analysis}

Throughout the study we have assumed that the invasive measure represents the "true" or reference value; hence the difference between the noninvasive and the invasive measure gives the measurement error. 
The Wilcoxon signed rank test was used to compare measurements. A p $<0.05$ was considered statistically significant.

Results are expressed as median (interquartile range).

\section{Results}

Due to a large number of ectopic beats, 3 patients had to be excluded from analysis. A representative example of simultaneous recordings of invasive and Finometer SAP and DAP in a post-MI patient is shown in Figure 1. The corresponding autospectra, and the coherence function for SAP time series are shown in Figure 2.

Results for SAP and DAP spectral measurements, expressed as median (interquartile range), are summarized in Table 1a and $1 \mathrm{~b}$ respectively.

Summary results for the coherence and for the transfer function modulus between SAP and RR are given in Table 2 for both invasive and Finometer measurements.

Table 1a. Summary results for SAP spectral measurements

\begin{tabular}{ccccc}
\cline { 2 - 5 } & VLF & LF & HF & TP \\
\hline $\begin{array}{c}\text { Inv power } \\
\left(\mathrm{mmHg}^{2}\right)\end{array}$ & $1.9(1.2)$ & $1.0(0.9)$ & $1.6(2.7)$ & $6.8(5.4)$ \\
\hline $\begin{array}{c}\text { Fin power } \\
\left(\mathrm{mmHg}^{2}\right)\end{array}$ & $5.5(4.4) \dagger$ & $1.8(1.7) \ddagger$ & $1.1(1.7) \ddagger$ & $12.5(7.9) \dagger$ \\
\hline $\begin{array}{c}\text { Error } \\
(\%)\end{array}$ & $139(174)$ & $83(105)$ & $-28(50)$ & $60(110)$ \\
\hline $\begin{array}{c}\text { COP } \\
(\%)\end{array}$ & $83(28)$ & $82(18)$ & $92(20)$ & $79(17)$ \\
\hline$\dagger \mathrm{p}<0.001 ; * \mathrm{p}<0.01$ in the comparison Inv Vs Fin
\end{tabular}

$\dagger: \mathrm{p}<0.001 ; \ddagger: \mathrm{p}<0.01$ in the comparison Inv Vs Fin

Table 1b. Summary results for DAP spectral measurements

\begin{tabular}{ccccc}
\cline { 2 - 5 } & VLF & LF & HF & TP \\
\hline $\begin{array}{c}\text { Inv power } \\
\left(\mathrm{mmHg}^{2}\right)\end{array}$ & $0.9(0.5)$ & $0.5(0.4)$ & $0.3(0.2)$ & $2.0(1.7)$ \\
\hline $\begin{array}{c}\text { Fin power } \\
\left(\mathrm{mmHg}^{2}\right)\end{array}$ & $1.4(1.5) \dagger$ & $0.6(0.8) \dagger$ & $0.2(0.2) \ddagger$ & $3.7(2.9) \dagger$ \\
\hline $\begin{array}{c}\text { Error } \\
(\%)\end{array}$ & $64(52)$ & $36(62)$ & $-16(35)$ & $53(44)$ \\
\hline $\begin{array}{c}\mathrm{COP} \\
(\%)\end{array}$ & $92(13)$ & $88(10)$ & $90(19)$ & $89(11)$ \\
\hline
\end{tabular}

$\dagger: p<0.005 ; \ddagger: p<0.05$ in the comparison Inv Vs Fin

Inv: invasive measurement, Fin: Finometer measurement,

Error: percentage error, COP: Coherent output power

\section{Discussion}

Our results show that the total variability of systolic blood pressure fluctuations is greatly enhanced by the
Finometer device. When total variability is partitioned into its contributions from the three bands analyzed, a significant increase is found in both VLF and LF bands, while respiratory frequency components (HF band) are significantly attenuated. The percentage error ranges from $+139 \%$ in the VLF band to $-28 \%$ in the HF band

Table 2. Summary results for average coherence and transfer function modulus between invasive SAP and RR and Finometer SAP and RR in the LF band.

\begin{tabular}{ccc} 
& MSC & TF $(\mathrm{ms} / \mathrm{mmHg})$ \\
\hline Inv & $0.36(0.18)$ & $9.4(5.9)$ \\
\hline Fin & $0.26(0.14) \dagger$ & $7.1(2.4) \dagger$ \\
\hline
\end{tabular}

Inv: invasive measurement, Fin: Finometer measurement MSC: magnitude square coherence, TF: transfer function modulus

$\dagger: \mathrm{p}<0.005$ in the comparison Inv Vs Fin

According to linear system analysis, we found that about $80 \%$ of Finometer power in VLF and LF bands is linearly related to the corresponding power in the invasive signal $(90 \%$ in HF band), limiting the contribution of noise and nonlinearities to about $20 \%$ (10\% in HF band) and indicating that the differences are mainly due to amplification or attenuation effects.

As regards diastolic pressure, most considerations done for systolic pressure hold, but the error was much lower, ranging from $+64 \%$ in the VLF band to $-16 \%$ in the HF band.

The results we found in the analysis of coherence and transfer function between SAP and RR time series are quite interesting, indicating that in the LF band the invasive pressure signal has a stronger linear relationship with the RR interval than the Finometer signal and that in the computation of baroreflex sensitivity, a significant and relevant underestimation occurs when using the noninvasive pressure signal.

Several factors could contribute to the differences we found: inaccuracies in the invasive measurements, the different site of pressure measurements, interference from the vasomotor activity of small finger arteries. We hypothesize that the latter phenomenon could play a major role, since the volume-clamp method used by the Finometer assumes a pure elastic behaviour of the walls of small arteries under the cuff and the time varying smooth muscle forces acting on the walls of the clamped arteries can interfere with the measurements of finger arterial pressure causing a time-varying bias.

Our findings substantially confirm those previously obtained with the Finapres device. This is not surprising since the Finometer is largely based on the same 
technology, and most improvements are in the software which processes the pressure signal in an attempt to reconstruct the brachial artery waveform.

Unfortunately, however, the reconstructed waveform is not yet accessible as an analog output signal in the devices currently on the market.
[4] Task Force of the European Society of Cardiology and the North American Society of Pacing and Electrophysiology. Heart rate variability. Standards of measurement, physiological interpretation and clinical use. Circulation 1996; 93 1043-1065

[5] Pinna GD, Maestri R, Raczak G, La Rovere MT. Measuring baroreflex sensitivity from the gain function between arterial pressure and heart period. Clin Sci (Lond) 2002; 103(1): 81-88

\section{References}

[1] Pinna GD, Maestri R, Mortara A. Estimation of arterial blood pressure variability by spectral analysis: comparison between finapres and invasive measurements. Physiol Meas 1996; 17(3): 147-169

[2] Omboni S, Parati G, Frattola A, Mutti E, Di Rienzo M Castiglioni P, Mancia G. Spectral and sequence analysis of finger blood pressure variability. Comparison with analysis of intra-arterial recordings. Hypertension 1993; 22: 26-33

[3] Pinna GD, Maestri R, Di Cesare A. Application of time series spectral analysis theory: analysis of cardiovascular variability signals. Med \& Biol Eng \& Comput 1996; 34: $142-148$
Address for correspondence.

Ing. Roberto Maestri

Department of Biomedical Engineering,

S. Maugeri Foundation, Scientific Institute of Montescano. 27040 Montescano (PV) Italy.
E-mail: rmaestri@fsm.it 